\title{
Das Werk neben dem Werk: Max Weber in seinen Briefen
}

\author{
Hans-Peter Müller
}

Angenommen: 17. März 2021 / Online publiziert: 13. April 2021

(C) Der/die Autor(en) 2021

Max Weber, Reisebriefe 1877-1914. Herausgegeben von Rita Aldenhoff-Hübinger und Edith Hanke. Mit einem Einleitungsessay von Hinnerk Bruhns. Tübingen: Mohr Siebeck 2019.

Max Weber, Gelehrtenbriefe 1878-1920. Herausgegeben von Rita Aldenhoff-Hübinger und Edith Hanke. Mit einem Einleitungsessay von Gangolf Hübinger. Tübingen: Mohr Siebeck 2020.

\section{Einleitung}

Pünktlich zum hundertsten Todestag von Max Weber am 14. Juni 2020 ist die Max Weber-Gesamtausgabe (MWG) fertig geworden. In 47 Bänden, die sich wegen der Halbbände auf insgesamt 54 hochschrauben, haben wir jetzt den ,ganzen“ Weber vor uns - zumindest soweit schriftliche Zeugnisse von ihm überliefert sind. Und was sehen wir da? Ein unglaubliches Werk, vielschichtig, komplex und kompliziert, thematisch ganz breit aufgestellt und die gesamte Menschheitsgeschichte umfassend. Weber ist keineswegs nur Soziologe, sondern umgreift die Kultur-, Religions-, Wirtschafts-, Rechts-, Geschichts-, Politik- und Sozialwissenschaften. Das erklärt seine große Anschlussfähigkeit. Und doch ist das „Ganze“ ein riesiger Torso voll von genialen Skizzen in systematischer Absicht, die paradigmatisch und exemplarisch zeigen sollen, wie eine „Soziologie“ als verstehende Wissenschaft möglich werden könnte. Es ist dieser Charme der Unabgeschlossenheit und des Anfangs,

H.-P. Müller $(\bowtie)$

Institut für Sozialwissenschaften, Humboldt-Universität zu Berlin, Universitätsstr. 3b, 10117 Berlin, Deutschland

E-Mail: hpmueller@sowi.hu-berlin.de 
der heute noch besticht und dafür sorgt, dass die Soziologie immer wieder vom Nimbus ewiger Jugendlichkeit zu zehren vermag. Das gilt auch für die Weber-Rezeption selbst: Sicher - noch nie haben wir so viel gewusst über einen der größten Soziologen. Und doch: Die MWG lehrt Demut und eröffnet die Möglichkeit eines Neuanfangs. Im 21. Jahrhundert werden wir Weber neu lesen und - ohne den myriadenhaften und verdienstvollen Bemühungen der vergangenen Sekundärliteratur Unrecht tun $\mathrm{zu}$ wollen - mit noch größerem Verständnis und vertiefter Einsicht. Genau diese Chance eröffnet die MWG, die editorische Standards zu setzen vermag und uns einen vorzüglich annotierten wie kommentierten Max Weber zugänglich macht. Auch hier marschieren Marx und Weber - als die global bekanntesten Soziologen überhaupt (Beytía und Müller 2019) - Arm in Arm: Marx wird ebenfalls neu zu lesen sein, wenn denn die MEGA dereinst fertig werden wird.

Die MWG ist in drei Abteilungen aufgeteilt: Die erste umfasst ,Schriften und Reden“, die sich auf 25 Bände belaufen. Rechnet man mit ein, dass u. a. Wirtschaft und Gesellschaft aufgespalten worden ist, in Band 23 für den noch von Weber selbst in den Druck gegebenen Teil und die älteren Manuskripte als ,Die Wirtschaft und die gesellschaftlichen Ordnungen und Mächte“, Band 22 in fünf Teilbänden, dann summiert sich dieser Teil des Werks auf 33 (Teil-)Bände. Man könnte meinen, es sei kein Zufall gewesen, dass auch hier Marx und Weber editionspolitisch geistig vereint worden sind und ihre jeweiligen Hauptwerke die gleiche Bandzahl tragen. In der MEW ist Band 23 Das Kapital, Bd. 1, in der MWG Wirtschaft und Gesellschaft.

Die dritte Abteilung der MWG enthält in sieben Bänden Webers Vorlesungen, entweder in Form seiner eigenen Manuskripte oder als Mit- und Nachschriften: von der ,Allgemeinen (,theoretischen“) Nationalökonomie von 1894-1898“ bis zur ,Allgemeinen Staatslehre und Politik (Staatssoziologie)“. Was indes erstmalig in Gänze präsentiert wird, sind die „Briefe“ als zweite Abteilung der MWG. Sie umfassen elf Bände von den Jugendbriefen bis zu den Nachträgen und einem Gesamtregister.

Max Weber war ein veritabler Briefeschreiber. Mehr als 3500 Briefe sind allein in den elf Bänden versammelt. Ein Wermutstropfen freilich ist die Preisgestaltung der sehr gut ausgestatteten Bücher. Nur wohlhabende Weber-Enthusiasten werden pro Band circa dreihundert Euro aufbringen und für den Gesamtkorpus über dreitausend Euro anlegen wollen. Während die Schriften durch die preisgünstige Studienausgabe einer breiten Leserschaft zugänglich gemacht werden, gilt das für das Briefwerk bislang (noch) nicht. Insofern wird der Bibliotheksbesuch unumgänglich, um die elf Bände eingehend studieren zu können. Das ist bedauerlich, denn das Briefwerk eröffnet nochmals einen ganz neuen Blick auf den uns bekannten Weber. Tatsächlich ist es nicht übertrieben, von einem „Werk neben dem Werk“ (Lepsius 2016) zu sprechen. Aus der Not eine Tugend machend, haben die Mitherausgeberinnen des Briefwerkes, Rita Aldenhoff-Hübinger und Edith Hanke, nun zwei Auswahlbände vorgelegt, die zum einen die „Reisebriefe 1877-1914“ und zum anderen die „Gelehrtenbriefe 1878-1920“ von Weber vorstellen.

Um es gleich vorwegzunehmen: Diese gelungene Zusammenstellung vermittelt einen ersten Eindruck von Max Weber, der Person in ihrer Vielschichtigkeit als Privatmensch, Gelehrter und Politiker. Und was das Wichtigste ist: Der „Mythos von Heidelberg", mit dem streng asketischen Blick, wie er uns aus den wenigen Fotografien bekannt geworden ist, wird plötzlich lebendig. Neben dem sachlichen, 
nüchternen und rechtschaffenen Wissenschaftler, der die Peitsche der „Werturteilsfreiheit" mit tiefem Ernst schwingt, lernen wir den familiären und politischen Weber kennen. Während sein Werk vor begrifflicher Tiefenschärfe und penibler historischer Empirie seine Leserschaft zuweilen vor große Herausforderungen des Verständnisses stellt, offenbaren die Briefe einen ebenso klugen wie warmherzigen Beobachter des Menschenverkehrs, dem im Übrigen nichts Menschliches fremd war. Und noch etwas: Der Streitpunkt, ab wann denn nun Weber „eigentlich“ ein Soziologe geworden sei, ob vor seinem krankheitsbedingten Zusammenbruch von 1898 oder erst nach seiner allmählichen Genesung 1903/4, könnte nun entschieden sein. Denn Max Weber ist ein soziologisches Naturtalent, ein genauer Beobachter des Soziallebens und der Verhaltensweisen seiner Mitmenschen. Schon in ganz jungen Jahren zeigt sich seine unerschütterliche Urteilskraft. Von Weber lernen, heißt soziologisch sehen und erkennen lernen. Das lässt sich an seinen Reisebriefen, aber auch an seinen Gelehrtenbriefen zeigen.

\section{Webers Reisebriefe (1877-1914)}

Die Reisebriefe haben die Herausgeberinnen in sieben Abschnitte unterteilt, die von den ersten Reisen (1877-1880) über die Hochzeitsreise (1893), die Reisen nach Schottland und Irland (1895), nach Frankreich und Spanien (1897), Amerika (1904), den Niederlanden und Belgien (1903 und 1907) bis zu seinen Besuchen in Südfrankreich, Italien und Ascona (1902-1914) reichen. Schon Goethes „Wilhelm Meister“ hatte demonstriert, dass neben dem Buchwissen auch Erfahrungswissen zählt, das nur gewinnt, wer mobil ist, statt sein Leben lang auf dem gleichen Fleck zu verharren wie Kant in Königsberg. Nicht nur Lektüre, sondern auch Reisen bildet und formt Welthaltigkeit. Max Weber senior, von der biographischen Sekundärliteratur meist ungebührlich geschmäht, beginnt 1877, mit seinen beiden älteren Söhnen Max und Alfred auf Wander- und Kulturreisen zu gehen. Max als ältestem Sohn obliegt die Berichterstattungspflicht an die Mutter Helene daheim. Die doppelte Reisetätigkeit muss man ernst nehmen: Es wird gewandert und wandernd wird Kultur erfahren, um anschließend brieflich verarbeitet zu werden. So berichtet er 1877 nach Hause ,,von dem Bismarck-Denkmal auf dem Burgberge bei Harzburg mit der Aufschrift ,Nach Kanossa gehen wir nicht!!“", um dann radebrechend dichtend zu enden: „Fremdling, kommst Du nach Berlin einst, melde, daß Du hier gesehn./Kirschen essend auf der Straße die Familie Weber gehn.“ (Weber 2019, S. 8) Mit sechzehn Jahren macht er seine erste Reise allein durch Schlesien und Böhmen und berichtet pflichtschuldigst von seinen akademischen Geselligkeitsobligationen. In Breslau trifft er die Diltheys: „Gegen Abend war ich bei Diltheys, wurde dessen Frau und Schwiegermutter vorgestellt und fuhr per Dampfschiff mit ihnen gemeinsam nach dem ,Oderschlösschen “ hinaus. Nach einem nochmaligen Abendessen, wobei viel von Herrn Prof. Mommsen und dessen Schwefelbande von Söhnen die Rede war, fuhren wir zurück." (S. 15) So lernt der junge Weber bereits frühzeitig den Umgang mit den bildungsbürgerlichen Größen, die ihn dereinst ausbilden sollten.

Im Jahre 1893, in dem Max Weber Marianne Schnitger geheiratet hat, erklingt schon ein ganz anderer Ton von einem fast gehetzten Ehemann. In seiner neuen 
Rolle noch völlig ungewohnt, sucht Weber seiner Frau mit einem Besuch in London, auf der Isle of Wight und schließlich in Paris standesgemäß etwas zu bieten. Man ist fast geneigt, von Reisestress zu sprechen, aber für das junge Paar scheint es nicht genug Abwechslung in kurzer Zeit gegeben zu haben. Weber berichtet stolz seiner Mutter aus Paris: „Ich glaube, daß nicht viele Hochzeitsreisepaare so viel in Bewegung auf allen denkbaren Vehikeln (und übrigens auch zu Fuß) gewesen sind als wir. Westminster, British Museum, St. Pauls, National Gallery, Tower, die Fleet Street Restaurants etc wurden in London mit Underground, Dampfer, Hansam und Omnibus angelaufen, ohne daß wir doch jemals das Gefühl gehabt hätten uns zu hetzen.“ (S. 24f.) Zu erotischen Verwicklungen scheint es dagegen weniger gekommen zu sein, denn nachdem sich die beiden in Paris eine leichte Erkältung zugezogen haben, berichtet Max seiner Mutter: ,wir husten beide etwas und wenigstens sie ist es Nachts noch nicht gewohnt, daß neben ihr Jemand athmet und hustet." (S. 26)

Auch bei ihrem Besuch in Schottland und Irland im Jahre 1895 gibt es wieder von einem Treffen unter Akademikern zu berichten. Denn auf dem Dampfer auf Loch Katrine entdeckt Weber ,das germanistische Bardengesicht Gierke's und dann die zierliche Tochter und die wie immer etwas schmuddelige Frau“. Mit Otto von Gierke und seiner Familie reist man ein Stück Weges, um dann den Schotten beim Abschied in Inversnaid ein denkwürdiges Erlebnis deutschen Barbarentums zu bescheren. Man hielt „,einen Lunch, an den die Kellner dort denken werden. Gierke begann ein Fressen, wie im Teutoburger Walde und ich machte nach. Die bestürzten waiters brachten, als stets Alles wieder verschwand, schließlich übermenschliche Quantitäten Roastbeefs, Salmen etc., vermutlich fürchtend, wir würden sonst nach den Menschen schnappen. Zu Dreien umstanden sie unseren Tisch, als aber Gierke, anstatt zwischen ,Marmalade‘ und ,Cheese` zu wählen, sich erst die erstere ,genehmigte' und dann auf denselben Teller Käse packte, öffnete sich der Mund des Chors und entsetzt starrten sie auf die Trümmer ihrer Habe, offenbar erleichtert, als endlich das Dampfschiff schellte und dem Fressen ein Ziel setzte.“ (S. 33f.) Die weiteren Reisebeobachtungen richten sich auf die agrarisch geprägte Landschaft, das miserable Essen und die kulturellen Gewohnheiten von Schotten und Iren. Aber natürlich kommt auch Weber, der Agrarhistoriker, auf seine Kosten, als er die schroffe soziale Ungleichheit der Landverteilung notiert. Nach einem Rundgang durch ein parkähnliches Castle auf den Hebriden notiert Weber: ,,als Professor fühlte man sich in seiner Tagelöhner-Existenz, und als Sozialpolitiker taxierte man den Besitzer als reif für den Dynamit.“ (S. 40)

Die nächste größere Reise machte das Paar nach Frankreich und Spanien 1897, wo sie sich mit Hilfe des Baedekers die Sehenswürdigkeiten von Südfrankreich und Nordspanien zu Gemüte führten. Weber, und hier scheint schon das religionssoziologische Interesse durch, lässt sich eine „Wunderheilung“ im Wallfahrtsort Lourdes nicht entgehen. Obwohl ,religiös unmusikalisch“, tritt selbst ihm unter dem Eindruck einer „gewaltige[n] Nervenerregung“ „einen Augenblick der kalte Schweiß auf die Stirn, und wie mag einem ,Gläubigen“ zu Muthe sein?" Weber beschreibt das Ritual der Heilung ganz genau, obwohl er die Heilung - das sog. „Magnificat“, „d.h. des Vorgangs, daß ein Kranker unter dem ungeheuren Druck der Aufregung plötzlich wie ein Rasender dem Bad entspringt: - ,je suis guéri!““ brüllt, nicht erleben konnte. Und doch: „Was ich an Kranken aus dem Baderaum humpeln sah, 
machte - soweit es eben auf seinen Füßen stehen konnte, - ganz vergnügte Gesichter, ich glaube einfach weil die Berührung mit dem hier auch psychisch unheimlich kalten Wasser und die ganz fürchterliche Exaltation überstanden war.“ (S. $71 \mathrm{f}$.)

In Bordeaux notiert Weber die Krise des Weinbaus durch den Reblausbefall mit der Folge wachsender Konzentration zu großen Gütern, denn die kleinen Bauern können sich die nötigen Mittel gegen die Reblaus nicht leisten. „Die großen Besitzer - so Rothschild, welcher Chateau Lafite besitzt - können es, aber die BordeauxWein-Cultur wird auf diese Art aus einer Massencultur ein Betrieb, der als Luxusgegenstand, ähnlich dem deutschen Rittergut, nicht um seiner wirtschaftlichen Rentabilität willen, von Millionären gehalten wird, und ein Produkt erzeugt, welches immer mehr nur dem Luxusconsum dient.“ (S. $75 \mathrm{f}$.)

In Spanien faszinieren Weber, den Agrarhistoriker, die spanischen Bauern: „Die stolze Freiheit des Wesens, die den spanischen Bauern im Norden und in der Mitte des Landes überhaupt auszeichnet, ist hier mit kaufmännischer Klugheit gepaart [...]. In den baskischen Provinzen beherrscht der Einzelhof die Landschaft, den Mittelpunkt auch des bäuerlichen Lebens bilden die zahlreichen kleinen, oft sehr kleinen Städte.“ (S. 79) Weber, den Genealogen des Kapitalismus, beeindruckt aber auch der boomende Eisenerzbau bei Bilbao. Nach einem gelungenen Abend ,im deutschen Club in Bilbao - Skat gespielt, bei Pilsner Bier, und mit dem Consul, bei dem ich vorher zu Abend gegessen und glänzende Moselweine [...] vorgesetzt erhalten hatte, nachdem er sich als Kartellbruder aus Tübingen entpuppt hatte“ (S. 80) - registriert Weber interessiert nicht nur ,kleine Brutstätten des Capitalismus“ wie Zementwerke, Maschinenfabriken, Stahlwerke, Eisenhütten, Möbelfabriken, unterstützt durch die elektrische Beleuchtung, ,von Siemens \& Halske (dessen Ingenieur ich in Bilbao kennenlernte) unter Ausnutzung der Wasserkraft" (S. 82). Vielmehr notiert er, dass „die Eisengruben hier, zur Zeit in dieser Art wohl die größten der Welt, [...] schon ganze Berge vollständig abgetragen“ (ebd.) hätten.

Neben der Landwirtschaft und der Industrialisierung hält er natürlich Einkehr bei der Religion und besucht das Kloster Loyola, ,,erbaut auf der Geburtsstätte des H1. Ignaz und alle Reminiscencen an denselben conservierend, neustens durch eine trotz des schwarzen Marmors im Innern zufolge ihrer zopfigen Überladenheit unschön wirkende Kirche, und im Übrigen jetzt Convikt“". (S. 83) Blühende Landwirtschaft und boomende Industrialisierung könnten eine großartige gesellschaftliche Entwicklung einleiten, wenn da nicht die spanische Politik und Verwaltung wären. Weber registriert ,den unerhörten Contrast zwischen der Tüchtigkeit der Landbevölkerung in diesen schönen Provinzen und der Niederträchtigkeit der spanischen Verwaltung [...]. Der Schuft fängt beim Beamten an.“(S. 86) Da ein allgemeines Steuersystem noch im Argen liegt, erfolgen die Einnahmen durch die Wahlen in der Demokratie. „Die geschonte Steuerkraft des Capitals wirkt so als ,Stimmkaufkraft“ bei den Wahlen, der Besitzende zahlt statt der Steuern Wahlbestechungsgelder.“ (S. 89) Ein solches System fördert die Korruption geradezu heraus. „Die gewaltige Macht der auf solchen Unterlagen ruhenden Capitalien [...] nutzt nun den trostlosen Zustand der spanischen Verwaltung in ungeheuerlichen Dimensionen aus. Die Bestechlichkeit des Gouverneurs, der Minister, aller staatlichen Beamten überhaupt ist den Herren, die ich hier sprach, schlechthin selbstverständlich.“ (S. 91) Weber, der sich mit der römischen Agrargeschichte beschäftigt hat, zieht sofort die Parallelen 
am Beispiel des staatlichen Monopols auf die Dynamitproduktion: „da wie im alten Rom die Erhebung aller Steuern verpachtet wird an private Unternehmer gegen feste Pachtsummen im Wege des Meistgebotes, so geschieht dies auch mit dem Betrieb der ,staatlich“ monopolisierten Dynamitfabrikation. Es wird zu Meistgeboten aufgefordert für die Conzession zur Dynamitproduktion. Natürlich läuft nur ein Gebot ein: das der schon bestehenden Fabriken, die sich zu einem Ring zusammengeschlossen haben. Sie zahlen also der Staatskasse einen festen Betrag jährlich dafür, daß sie das Recht haben, fortan allein, ohne Concurrenz, Dynamit zu fabrizieren, und kommen auf ihre Kosten für jene Abfindungssumme an den Staat, indem sie - wie es geschehen ist - den Preis auf das Doppelte erhöhen: das ist das Ergebnis des ,staatlichen “ Monopols.“ (S. $91 \mathrm{f}$.) Webers Fazit fällt eindeutig aus: „So knechtet der Übermuth des Kapitals in aller Form Rechtens den hülflosen Staat.“ (S. 92)

Die größte Reise stellt zweifellos die nach Amerika dar. Da sie vielfach in der Literatur (Offe 2004; Scaff 2013) kommentiert wurde, können wir uns kurzfassen. Im Jahre 1904 findet im Rahmen der Weltausstellung der ,World Congress for Arts and Sciences“ in St. Louis, Missouri, statt, und der wieder genesene Max Weber besorgt sich eine Einladung von Hugo Münsterberg (Harvard University), den er aus gemeinsamen Zeiten in Freiburg kennt. Weber trägt dort sein Lieblingsthema über Agrarprobleme vor - auf Deutsch, denn zu diesem Zeitpunkt werden die amerikanischen Ökonomen noch in Deutschland ausgebildet und Deutsch gilt als Wissenschaftssprache. Im Gegensatz zu seinen deutschen Kollegen, die die Vereinigten Staaten als „Kulturhölle“ (so sein Mitreisender Ernst Troeltsch) empfinden, ist Weber begeistert von der neuen Welt. Der wirtschaftliche Aufbruch, den er dort beobachtet, wird zu seinem eigenen Aufbruch nach seiner langen Krankheit seit 1898. Auch hier lässt sich sein unbestechliches soziologisches Auge bewundern, jetzt auch schon analytisch verfeinert durch die Anfänge seiner eigenen Soziologie. Am packendsten sind sicherlich Webers Schilderungen der unfassbaren Zustände in den ,Yards“, den Schlachthöfen von Chicago - die dann zwei Jahre später ein junger Journalist namens Upton Sinclair mit seinem Buch The Jungle (1906) aufdecken wird, was im gleichen Jahr zum Pure Food and Drug Act von Theodore Roosevelt führt. Einer zentralen Episode im „Rassenkampf“ an der Frontier wohnt Weber bei der Landverteilung in Oklahoma bei, die er als Tragödie schildert. „Heut sah ich die Indianer truppweise kommen ihr Geld zu holen - die Vollblutleute haben einen eignen müden Zug im Gesicht und sind sicherlich dem Untergang geweiht [...]. " (S. 121) Ihm ist klar, dass die alte Welt der Ureinwohner verschwunden sein würde, sollte er nochmals nach Amerika kommen. Weber sollte nicht, denn es ergab sich, trotz anderslautender Absichten, keine weitere Gelegenheit.

Die Niederlande und Belgien bereiste Weber 1903 und 1907. Die Attraktion dort war natürlich Rembrandt, hatte doch die Begeisterung für den holländischen Maler längst auch Deutschland erreicht. In kurios-konservativer Weise hatte Julius Langbehn (1890) mit seinem Bestseller Rembrandt als Erzieher den Namen des Malers für den deutschen Nationalismus usurpiert, was Georg Simmel (2003a [1890]), 
sonst stets in vornehmer Weise zurückhaltend, zu einer vernichtenden Rezension ${ }^{1}$ veranlasst hatte. Rembrandt wird dann bei Simmel (2003c [1916]) selbst zum Gegenstand eines seiner Künstler-Porträts - neben seinem Goethe (2003b [1912]) -, um an ihm das ,individuelle Gesetz“ und die gelungene ästhetische Lebensgestaltung zu demonstrieren. Auch Weber ist von Rembrandts Bildern so begeistert, dass er sich einen Kohledruck kauft. „Das Auge des Königs wirkt im Original mächtiger, Rembrandt malte es, als er nach Verlust seiner Saskia, seines Vermögens u. seiner Bilder, bankrott erklärt, aber auf der Höhe seines Könnens, einsam mit seinem Sohn u. seiner treuen Hendrikkie in Amsterdam lebend das Alter kommen fühlte. Dem Museumsdirektor, dessen Privatbesitz es ist, bot Pierpont Morgan (vom Steel-Trust) vergebens 500000 Gulden dafür.“(S. 155) So verbringt er viel Zeit im Rijksmuseum vor Rembrandts „Nachtwache“, geschult durch Carl Neumanns einschlägigen Rembrandt (1902). Auf Spinozas Spuren wandelnd, registriert er angenehm überrascht, dass der Philosoph ,,in der höfischen Kirche im Haag am vornehmsten Platz - unter der Kanzel, vis-à-vis der Hofloge, liegt, mit einer Gedenktafel (der einzigen) für ihn u. die s. Z. gemordeten Häupter der niederländischen Republik, Gebrüder de Witt, gemeinsam“. (S. $161 \mathrm{f}$.)

Die Reisen in den Süden nach Südfrankreich, Italien und Ascona (1902-1914) sind allesamt Fluchten aus dem Heidelberger Privatgelehrtenalltag. Weber hatte recht rasch wieder zu alter Arbeitswut gefunden, mit der Folge ständiger Überlastung und übermäßigen Tablettengebrauchs. Der Süden wird so zum Versprechen, die eigenen Nerven wieder in Ordnung zu bringen. So schreibt er im März 1908 aus Le Lavandou an Robert Michels: „Ich muß mich sehr mühsam über Wasser halten bei Entwöhnung von den Schlafmitteln, Trional, Bromidin etc. etc., die ich im Winter nur für den elenden Artikel ,Agrargeschichte, Altertum' im Handwörterbuch der Staatswissenschaften brauchte." (S. 184) Nun, kein Wunder die Überanstrengung, hatte Weber doch die Aufforderung der Herausgeber, seine 15 Seiten aus der Erstauflage auf 8 Seiten für die Neuauflage zu kürzen, wohl etwas falsch verstanden: Am Ende kamen bei der Überarbeitung fast 300 Buchseiten heraus, und die Agrarverhältnisse im Altertum (1909) wurden ,eingeleitet“ durch eine auch heute noch unübertroffene Theorie der antiken Staatenwelt (Weber 1924).

1913/1914 zieht es Weber nach Ascona, neugierig auf das Studium der neuen alternativen Lebensformen auf dem Monte Verità. Gleichzeitig unterstützte er in schwierigen Rechtsfragen Gräfin Franziska zu Reventlow und Frieda Gross, die Ehefrau des Psychoanalytikers Otto Gross. Auf dem Rückweg über Zürich mit Mina Tobler, seiner Klavierlehrerin und Geliebten, teilt er seiner Frau mit, wie froh er sei, „diese Welt voller Zauberweiber, Anmuth, Tücke und Glücksbegier“ hinter sich zu lassen. „Ich muß ja sagen: zwischen diese schönen, in gewissem Sinn auch doch ,menschlichen', aber hintergrundlosen Eindrücke einer nur auf Sensation gestellten Welt war es eine Art von Oase der ,Reinheit ' [...] hier gestern diese Fahrt nach der

\footnotetext{
1 Nachdem Simmel die ,gewagteste Analogiespielerei“ und die „direkt unsittlich“ zu nennende „Art der Beweisführung“ kritisiert hatte, resümiert er „die Kapuzinade des Verfassers“: „Als eine Aeußerung dieses Zeichens der Zeit soll an dieser Stelle das vorliegende Buch registrirt werden, in dem die sprühende Geistreichheit der Form mit der Unklarheit und Unbedeutsamkeit des Inhalts den denkbar größten Kontrast bildet.“ (Simmel 2003a [1890], S. 241 ff.)
} 
Ufenau mit dem so viel weniger verschwenderisch ausgestatteten, aber in seiner distanteren und zart schwärmerischen Art doch ,nobler" wirkenden Kind.“ (S. 208) Der Ausbruch des Ersten Weltkrieges im August 1914 sorgte dafür, dass Weber seine empirischen Feldbeobachtungen alternativer Lebensgestaltung und erotischer Lebensführung nicht fortsetzen können sollte. Mit dem Reisen war auch erst einmal Schluss.

\section{Webers Gelehrtenbriefe (1878-1920)}

Waren die Reisebriefe in erster Linie für den inneren Kreis der Familie und nicht für eine breitere Öffentlichkeit bestimmt, so gilt das für die Gelehrtenbriefe in dieser strikten Weise nicht. Denn sie sind an die Adresse der Kollegenschaft wie auch an politische Kreise gerichtet. Allerdings haben die Herausgeberinnen auch hier eine Einteilung in drei Rubriken vorgenommen: Lebensführung (1878-1920), Politik (1878-1920) und Wissenschaft (1891-1920). Die erste Rubrik beleuchtet markante biographische Stationen von Webers Leben. So berichtet er seinen Eltern vom Studium, belehrt seinen jüngeren Bruder Alfred anlässlich dessen Konfirmation über seine Christenpflichten, löst seine Verlobung mit Emmy Baumgarten, um frei zu sein für eine Heirat mit Marianne Schnitger, schreibt Letzterer einen emphatischen Brautbrief, der gleichwohl auf eine Gefährtenehe ohne erotische Lebensführung vorbereitet, berichtet dem Bruder Alfred von seinem Bruch mit dem Vater, diskutiert mit Robert Michels in eroticis und beichtet Ferdinand Tönnies, der ,,alle Theologie als ,Unsinn““ verwirft (Weber 2020, S. 55), dass er, obschon selbst ,religiös ,unmusikalisch““(S. 56), viel Verständnis für die aufrichtig um ihren Glauben kämpfenden Menschen aufbringt. Die großen Themen sind natürlich seine Krankheit, die Liebe, der Ehebruch, der Beruf des Gelehrten und der Tod. Auch wenn Weber nach der langen Phase seines Zusammenbruchs den Beruf des Privatgelehrten wieder aufnimmt, schwebt doch seine Krankheit wie ein Damoklesschwert ständig über ihm. Gegenüber seinem Heidelberger Kollegen Georg Jellinek, der über seine eigenen Beschwerden eine Brücke der Solidarität zu Weber in aufmunternder Absicht zu bauen hoffte, verbittet er sich diese Art von Zuspruch, so als ob er als „,Malade imaginaire“" (S. 54) zu gelten habe. Mina Tobler gegenüber beklagt er sich über die Grenzen seiner Schaffenskraft, ,,und ,das andre Ufer" mit seiner gewissen Einsamkeit gegenüber allen Gesunden, auch den Nächststehenden, ist mir ja vertraut.“ (S. 73)

Der erotische Diskurs über die freie Liebe, der vor der Wende zum 20. Jahrhundert einsetzt, sollte auch das beschauliche Heidelberg und sein gesittetes Bildungsbürgertum erreichen. Der charismatische Psychiater Otto Gross nimmt Heidelberg im Sturm und versucht, Frauen dadurch zu emanzipieren, dass er ihnen ein Kind macht - so auch Else von Richthofen. Die Webers sind darüber recht erschrocken, aber Max Weber übernimmt die Patenschaft für den kleinen Peter, der aus der Liaison zwischen Gross und Else erwachsen war. Dass aber nun auch noch ein Aufsatz von Gross im Archiv erscheinen sollte, das war zu viel für Weber, und er rechnet mit der Psychoanalyse von Freud und deren Adaption durch Gross gnadenlos ab. Die neue Sexualethik ,muß überhaupt den Muth haben, mir zu empfehlen, jeder 
noch so hündisch gemeinen Regung meiner Begierden und meines Trieblebens zum ,Abreagieren', - und das heißt: zu einer ihr irgendwie adäquaten Form der Befriedigung, - freie Bahn zu geben, weil andernfalls meine lieben Nerven Schaden nehmen könnten: der echte - und wohlbekannte - Standpunkt des medizinischen Banausen!“” (S. 48) Dem „Ideal“ dieser Ethik vermag der kranke Weber wenig abzugewinnen: „der normale Gesundheitsprotz und ärztlich controllierte Philister der Makrobiotik“ (S. 51). Und doch entfaltet der erotische Diskurs seine Wirkung. Weber selbst beginnt eine Liebschaft mit Mina Tobler und tröstet Emil Lask, der unter seinem Ehebruch mit Lina Radbruch, der Ehefrau von Gustav Radbruch, leidet. ,Was geschehen ist, ist geschehen, und es handelt sich darum, allmählich allen Beteiligten zu einem menschlich abschließenden Verstehen: - wie das Leben mit uns spielt - zu helfen. Das wird schon kommen und gelingen. Im Übrigen aber kann Schuld eine Kraftquelle werden, oder nicht, je nachdem wie man sie nimmt. Es wäre schlimm, wenn nur das ,Integer vitae " uns zu Vollmenschen machte - und nicht auch das, richtig genommene, Gegenteil. Dann hätte jedenfalls ich auf volles Menschentum verzichten müssen." (S. 69f.) Das musste er auch deshalb nicht, weil sich die Liebe seines Lebens schließlich doch noch erfüllen sollte: 1919 beginnt er eine Affäre mit Else Jaffé. Die beiden hatten sich über den frühzeitigen Tod ihres Sohnes Peter, für den Weber die Patenschaft übernommen hatte, wieder angenähert. Die in diesem Zusammenhang entstandenen intimen Briefe waren weder für Webers Ehefrau noch seine Mutter oder seinen Bruder Alfred, der selbst mit Else liiert war, bestimmt, noch weniger indes für die Öffentlichkeit. Ihr Pathos ist Beleg für die Kraft der Liebe, die selbst den nicht nur religiös, sondern auch erotisch eher unmusikalischen Weber aus der Reserve locken konnte (Lepsius 2016).

Eigentlich wollte Weber eher eine praktische Stelle ergattern, als Gelehrter zu werden - wie seine fehlgeschlagene Bewerbung als Syndikus in Bremen (S. 112f.) zeigt. „Ein eigentlicher Gelehrter [...] bin ich nun einmal nicht“ (S. 25), gesteht er Emmy Baumgarten noch 1892. Im Jahre 1920 wird er dagegen seinen Austritt aus der Deutschen Demokratischen Partei (DDP) dem Vorsitzenden Carl Petersen damit begründen, dass er nun einmal Gelehrter geworden sei. „Der Politiker soll und $m u \beta$ Kompromisse schließen. Aber ich bin von Beruf: Gelehrter. [...] Der Gelehrte darf keine Kompromisse schließen und ,Unsinn“ [hier die Sozialisierungspläne, H.-P. M.] nicht, decken ‘. [...] Ich würde als Verbrecher an meinem Beruf handeln.“ (S. 161)

Der Tod hält - als vermeintlicher „Heldentod“ - im Gefolge des Ersten Weltkriegs kräftig Einzug, und Weber spendet einfühlsam und höchst persönlich angesichts solch furchtbarer Schicksalsschläge den betroffenen Familien Trost. Auch für den Freitod bringt er viel Verständnis auf, wie sein Brief an Friedrich Gundolf, dessen Vater Selbstmord begangen hatte, zeigt. Sein Fazit aus eigener Erfahrung: „Es gibt Nervenzustände, welche dem Kranken die eigne Existenz so vollkommen sinnlos erscheinen lassen, daß er die berechtigte Meinung haben kann, seinen Angehörigen den aus eignem Entschluß erfolgenden Abschied zumuthen zu dürfen, so unendlich schmerzlich ihnen dies fallen muß." (S. 63)

Wie sehr Weber ein durch und durch politischer Mensch war, zeigen die Briefe in der zweiten Rubrik. Sein Onkel Hermann Baumgarten, ein alter 48er, wird, im Gegensatz zum honoratiorenbürgerlichen Liberalismus seines Vaters, zum Ziehvater einer linksliberal kämpferischen Haltung, die freilich im rechten Kaiserreich stets 
minoritär bleiben muss. Weber arbeitet im Evangelisch-sozialen Kongress und wird zum politischen Berater und Unterstützer von Friedrich Naumann. Er verachtet den Antisemitismus in den Vorlesungen eines Heinrich von Treitschke, die regelmäßig „frenetischen Jubel“ (S. 108) unter seinen Zuhörern auslösen. Im September 1906 nimmt Weber am Mannheimer Parteitag der Sozialdemokratischen Partei teil und wird zutiefst enttäuscht. ,Ich hörte bei Bebel u. Legien mindestens 10 Mal: ,unsre Schwäche“ betont. Dazu der ganze äußerst kleinbürgerliche Habitus, die vielen behäbigen Wirtsgesichter, die Schwunglosigkeit, ohne doch den Entschluß, nun die Consequenzen ,nach rechts“ zu ziehen, wenn der Weg ,nach links ‘ versperrt ist oder dafür gilt, - diese Herren schrecken Niemand mehr.“ (S. 123) Am schärfsten arbeitet er sich indes am Regime von Kaiser Wilhelm II. ab. „Das Maß von Verachtung, welches uns, als Nation, im Ausland (Italien, Amerika, überall!) nachgerade - mit Recht! das ist entscheidend - entgegengebracht wird, weil wir uns dieses Regime dieses Mannes ,gefallen lassen', ist nachgerade ein Faktor von erstklassiger ,weltpolitischer' Bedeutung für uns geworden. Kein Mann und keine Partei, die in irgend einem Sinne ,demokratische“ und zugleich ,nationalpolitische“ Ideale pflegt, darf die Verantwortung für dieses Regime, dessen Fortdauer unsere ganze Weltstellung mehr bedroht als alle Colonialprobleme irgend welcher Art, auf sich nehmen." (S. 125) Sein Ton wird sich im Ersten Weltkrieg noch einmal verschärfen angesichts der unrealistischen Kriegszielforderungen konservativer Kreise. Der verschärfte U-BootKrieg, so seine Befürchtung, wird die USA in den Krieg hineinziehen. ,In der Annahme, daß das Eingreifen Amerikas nicht gerade unseren Untergang, wohl aber den Verlust des Krieges und eine schwere Gefährdung der Stellung der Dynastie mit sich bringen könnte, weiß ich mich einig mit sachkundigen Herren, welche das Opfer des Intellekts auch gegenüber der begreiflichsten moralischen Entrüstung nicht zu bringen vermögen.“ (S. 140) Als es soweit ist und Deutschland den Krieg verloren hat, seine schlimmsten Befürchtungen sich also bewahrheitet haben, die große Überraschung: Weber gibt sich als ,absoluter Optimist“: „Wir fangen noch einmal, wie nach 1648 und 1807, von vorn an, das ist der einfache Sachverhalt. [...] Natürlich gebietet die Selbstzucht der Wahrhaftigkeit uns zu sagen: mit einer weltpolitischen Rolle Deutschlands ist es vorbei. Die , angelsächsische Weltherrschaft ${ }^{6}$ [...] ist Thatsache. Sie ist höchst unerfreulich, aber: viel Schlimmeres - Russische Knute! haben wir abgewendet. Dieser Ruhm bleibt uns. Amerikas Weltherrschaft war so unabwendbar wie in der Antike die Roms nach dem 2. punischen Krieg, hoffentlich bleibt es dabei, daß sie nicht mit Rußland geteilt wird. Dies ist für mich Ziel unserer künftigen Weltpolitik, denn die russische Gefahr ist nur für jetzt, nicht für immer, beschworen. Im Augenblick ist natürlich der hysterische ekelhafte Haß der Franzosen die Hauptgefahr.“ (S. 150f.)

Die Briefe zur Rubrik ,Wissenschaft“ drehen sich vor allem um die Weber'schen Rufe nach Freiburg und Heidelberg, die Übernahme des Archivs für Sozialwissenschaft und Sozialpolitik mit Edgar Jaffé und Werner Sombart, die Gründung der Deutschen Gesellschaft für Soziologie und die Verlagskorrespondenz mit Paul Siebeck. Aber wer hätte gedacht, dass Weber beinahe zum wissenschaftlichen Biographen geworden wäre? So hatte er sich bereiterklärt, in der von Otto Hintze und Gustav Schmoller geplanten Reihe „Politiker und Nationalökonomen“ einen Band zu Rudolf von Gneist zu übernehmen. Weber hatte daran gereizt, „,die politische 
Geschichte der in der Hauptsache durch die nationalliberale und die ihr nahestehenden Parteien repräsentierten Mitarbeit des Bürgertums an der Politik, Genesis und Niedergang der specifisch bürgerlichen Epoche unserer Politik 1867-78 in einer Skizze darzustellen." (S. 172) Freilich wären da andere Personen zu behandeln gewesen, die Weber schon mit Hermann Baumgarten diskutiert hatte: Eduard Lasker, Rudolf von Bennigsen, Johannes von Miquel. Gneist hingegen gehörte für ihn zu ,den fatalsten und antipathischsten“ „,politische[n] Persönlichkeit[en]“, wenn es um ,den Niedergang der bürgerlich-politischen Entwicklung“ (ebd.) ging. Nicht nur die Gneist-Biographie blieb somit ungeschrieben, sondern auch die Lassalle-Biographie, die Schmoller ebenfalls aus Webers Feder haben wollte. Hier ist sich dieser nicht sicher, ob er ,gegenüber der tendenziösen aber in ihrer Art doch ausgezeichneten Bernsteinschen Arbeit etwas Neues zu leisten“ vermag. Außerdem zweifelt er daran, „ob ich für biographische Zwecke über so lebhafte Farben verfüge, wie sie bei Lassalle angebracht sind.“ (S. 175) Ein reizvoller Gedanke, sich vorzustellen, eine Biographie über Lassalle von Weber lesen zu können. An der Farbigkeit seiner Schilderung hätte es sicherlich nicht gemangelt, wie sein Briefwerk anschaulich demonstriert.

Weber stürzt sich mit großem Eifer in die Ausgestaltung des Archivs. Dort erscheinen nicht nur gleich sein „Objektivitätsaufsatz“ und seine „Protestantische Ethik“, sondern er versucht auch ausländische Autoren zu gewinnen. So hätte er gern von W.E.B. Du Bois, der an der Friedrich-Wilhelms-Universität in Berlin studiert und an der Harvard University als erster Schwarzer promoviert hatte, einen Aufsatz zum Verhältnis von „Rassen-“ und „Klassenfrage“ in den Vereinigten Staaten gehabt. Du Bois“ (1899) empirische Studie The Philadelphia Negro hatte Weber dermaßen beeindruckt, dass er Else Jaffé mit der Übersetzung beauftragt. Freilich brachte sie das Projekt nie zu Ende - angeblich wegen des schwierigen Englisch von Du Bois (Müller 2004). Alexander A. Tschuprow möchte er für einen Artikel über ,die Entwicklung des russischen sozialwissenschaftlichen Denkens“ (S. 193) gewinnen. Friedrich Gottl soll am Archiv mitarbeiten, und Weber macht ihn sogleich mit dessen editorischen Standards rückhaltloser Kritik bekannt: „Bitte polemisieren Sie so scharf wie möglich gegen meine Ansichten an den Punkten, wo wir differieren.“ (S. 194)

Groß ist auch sein Einsatz bei der Gründung der Deutschen Gesellschaft für Soziologie. Er tritt dem Vorstand als Rechner bei, ist aber schon nach dem ersten Soziologentag im Jahre 1910 restlos bedient. Einen Anspruch auf Wissenschaftlichkeit könne die Soziologie nur erheben, so Webers eherne Losung, wenn sie dem von ihm formulierten Grundsatz der Werturteilsfreiheit folgen würde. Als sich das nicht durchsetzen lässt, verlässt er 1911 den Vorstand und tritt 1914 endgültig aus der DGS aus. An Franz Eulenburg schreibt er frustriert: „Denken Sie doch nicht, daß Sie der Einzige wären, dessen Name Leute abstößt. Simmel, Tönnies, Sombart, ich, doch noch weit mehr. Aber das Schlimme ist: alle diese ,Refusés ' (ich gehöre ja auch dazu, denn daß ich es einmal bis zum Ordinarius gebracht hatte, kann mir doch nicht zeitlebens nachgetragen werden!) vertragen sich ja nicht, gönnen sich ja gegenseitig noch weniger als die Ordinarien, sind zu sachlichen Opfern ganz unfähig. [...] Ich muß jetzt an das wissenschaftliche Arbeiten zurück, so geht es nicht weiter, da ich der Einzige geblieben bin, der das Opfer seiner wissenschaftli- 
chen Individualinteressen gebracht hat - und damit doch nichts erreicht hat, als eine leerlaufende Maschine notdürftig in Gang zu halten." (S. $207 \mathrm{f}$.)

Es sind zwei wissenschaftliche Großprojekte, die Weber in der Folgezeit in Angriff nehmen wird: Die vergleichenden Analysen zu den Weltreligionen, um die „Protestantische Ethik“ einzubetten, sowie das Handbuch Grundriß der Sozialökonomik. 1913 kann Weber seinem Verleger stolz mitteilen, er habe ,eine geschlossene soziologische Theorie und Darstellung ausgearbeitet, welche alle großen Gemeinschaftsformen zur Wirtschaft in Beziehung setzt: von der Familie und Hausgemeinschaft zum ,Betrieb“, zur Sippe, zur ethnischen Gemeinschaft, zur Religion (alle großen Religionen der Erde umfassend: Soziologie der Erlösungslehren und der religiösen Ethiken, - was Tröltsch gemacht hat, jetzt für alle Religionen, nur wesentlich knapper), endlich eine umfassende soziologische Staats- und HerrschaftsLehre. Ich darf behaupten, daß es noch nichts dergleichen giebt, auch kein ,Vorbild “.” (S. 219) Wie recht Weber mit dieser Einschätzung hatte, zeigt die jetzt vorliegende Gesamtausgabe. Tatsächlich ist bis auf den heutigen Tag nichts Vergleichbares geleistet worden. In einer Fußnote fügt Weber noch hinzu: ,Später hoffe ich Ihnen dann einmal eine Soziologie der Cultur-Inhalte (Kunst, Litteratur, Weltanschauung) zu liefern, außerhalb dieses Werkes oder als selbständigen Ergänzungsband.“(ebd.)

„Ein Werk neben dem Werk“ - so hatten wir die 11-bändige Briefausgabe bezeichnet. Tatsächlich gilt das - und das demonstriert auch die vorliegende Briefauswahl in zwei Bänden - in zweierlei Weise. Die Briefe sind zum einen unerlässlich, um das torsohafte Gesamtwerk in Intention und Stoßrichtung besser zu verstehen. Sie sind wie ein Kommentar zu lesen. Zum anderen öffnen sie den Kosmos des Weber'schen Denkens, indem sie seine Netzwerke und Verbindungen in Familie, Verwandtschaft, Wissenschaft und Politik beleuchten. Webers Werk erscheint uns heute - ferngerückt durch ein Jahrhundert - wie ein eigenartiger und einzigartiger Solitär - und ein Stück weit stimmt dieser Eindruck ja auch. Aber das Briefwerk ermöglicht uns einen Zugang zu An-, Ein- und Aussichten, die ob ihrer subjektiven Färbung in seinem Werk so nicht zu finden sind. Die Stimme von Webers Werk wird besser verständlich durch die Stimmung seines Briefwerks. Insofern seien allen, die Webers verschlungenen Denkwegen auf die Spur kommen wollen, die beiden von Rita Aldenhoff-Hübinger und Edith Hanke sorgfältig edierten Bände nachdrücklich empfohlen. Eingeleitet werden sie jeweils durch einen klugen Einführungsessay von Hinnerk Bruhns (,„Er kann gar nicht genug Welt in sich schlingen“: Max Weber auf Reisen“) und Gangolf Hübinger (,Von Beruf: Gelehrter"). Da beide Bücher mit ausführlichen Verzeichnissen und einem Personenregister ausgestattet sind, lassen sich die abgedruckten Briefe im Briefwerk einwandfrei verorten. Das wird Studierenden wie Wissenschaftlern die eigene Arbeit erleichtern und werkkundig anleiten. Was das Wichtigste indes ist: Sie machen ihrerseits Lust auf Person und Werk, erschließen also auch dem Novizen Max Webers Denkwelt.

Funding Open Access funding enabled and organized by Projekt DEAL.

Open Access Dieser Artikel wird unter der Creative Commons Namensnennung 4.0 International Lizenz veröffentlicht, welche die Nutzung, Vervielfältigung, Bearbeitung, Verbreitung und Wiedergabe in jeglichem Medium und Format erlaubt, sofern Sie den/die ursprünglichen Autor(en) und die Quelle ord- 
nungsgemäß nennen, einen Link zur Creative Commons Lizenz beifügen und angeben, ob Änderungen vorgenommen wurden.

Die in diesem Artikel enthaltenen Bilder und sonstiges Drittmaterial unterliegen ebenfalls der genannten Creative Commons Lizenz, sofern sich aus der Abbildungslegende nichts anderes ergibt. Sofern das betreffende Material nicht unter der genannten Creative Commons Lizenz steht und die betreffende Handlung nicht nach gesetzlichen Vorschriften erlaubt ist, ist für die oben aufgeführten Weiterverwendungen des Materials die Einwilligung des jeweiligen Rechteinhabers einzuholen.

Weitere Details zur Lizenz entnehmen Sie bitte der Lizenzinformation auf http://creativecommons.org/ licenses/by/4.0/deed.de.

\section{Literatur}

Beytía, P., \& Müller, H.-P. (2019). Toward a digital reflexive sociology. Exploring the most globally disseminated sociologists on multilingual Wikipedia. Ms. Berlin.

Du Bois, W.E.B. (1899). The Philadelphia Negro. Philadelphia: The University of Pennsylvania Press.

Langbehn, J. (1890). Rembrandt als Erzieher. Leipzig: Hirschfeld.

Lepsius, M.R. (2016). Max Weber und seine Kreise. Tübingen: Mohr Siebeck.

Müller, H.-P. (2004). Das Gefühl, ein Problem zu sein. Über W.E.B. Du Bois. MERKUR, 58(660), 344-348.

Neumann, C. (1902). Rembrandt. Berlin: Speman.

Offe, C. (2004). Selbstbetrachtungen aus der Ferne. Tocqueville, Weber und Adorno in den Vereinigten Staaten. Frankfurt a. M.: Suhrkamp.

Scaff, L. A. (2013). Max Weber in Amerika. Mit einer Einführung von Hans-Peter Müller. Berlin: Duncker \& Humblot.

Simmel, G. (2003a) [1890]. „Rembrandt als Erzieher“. In G. Simmel, Gesamtausgabe, Bd. 1 (S. 232-242). Frankfurt a. M.: Suhrkamp.

Simmel, G. (2003b) [1912]. Goethe. In G. Simmel, Gesamtausgabe, Bd. 15 (S. 7-270). Frankfurt a. M.: Suhrkamp.

Simmel, G. (2003c) [1916]. Rembrandt. Ein kunstphilosophischer Versuch. In G. Simmel, Gesamtausgabe, Bd. 15 (S. 305-515). Frankfurt a. M.: Suhrkamp.

Sinclair, U. (1906). The Jungle. New York: Doubleday, Page \& Co.

Weber, M. (1924). Agrarverhältnisse im Altertum. In M. Weber, Gesammelte Aufsätze zur Sozial- und Wirtschaftsgeschichte (S. 1-288). Tübingen: Mohr Siebeck.

Weber, M. (2019). Reisebriefe 1877-1914. Herausgegeben von Rita Aldenhoff-Hübinger und Edith Hanke. Mit einem Einleitungsessay von Hinnerk Bruhns. Tübingen: Mohr Siebeck.

Weber, M. (2020). Gelehrtenbriefe 1878-1920. Herausgegeben von Rita Aldenhoff-Hübinger und Edith Hanke. Mit einem Einleitungsessay von Gangolf Hübinger. Tübingen: Mohr Siebeck.

Hans-Peter Müller geb. 1951. Prof. em. für Allgemeine Soziologie an der Humboldt-Universität zu Berlin. Forschungsschwerpunkte: Klassische und moderne Sozialtheorie, Sozialstruktur und soziale Ungleichheit, politische Soziologie und Kultursoziologie. Ausgewählte Veröffentlichungen: Pierre Bourdieu. Eine systematische Einführung, 2014; (mit T. Reitz, Hrsg.) Simmel-Handbuch, 2018; (mit S. Sigmund) Max Weber-Handbuch. Leben - Werk - Wirkung. 2. akt. u. erw. Aufl., 2020; Max Weber: Eine Spurensuche, 2020; Krise und Kritik. Klassiker der soziologischen Zeitdiagnose, 2021. 